\title{
Unilateral Maxillary Sinusitis Caused By Dental Bur, A case Report
}

\author{
Sahar Abdualkader Ismaeel \\ Saif Saadedeen Abdulrazaq \\ Department of Oral and Maxillofacial Surgery / College of Dentistry, University of Baghdad.
}

\begin{abstract}
the entrance of a foreign body (FB) into the maxillary antrum is common in trauma patients where bullets or any other materials trapped in the maxillary sinus; however, iatrogenic cause by a dentist that results in FB entrapment in the maxillary antrum is rare. All these FBs can cause sinusitis. If not treated properly they will cause serious infection, which may spread to the other paranasal sinuses. A careful clinical examination and treatment plan will prevent further complications. In this review, we present a rare case of dental bur (DB) found in the left maxillary antrum. A review of literature revealed only a few cases published which describe a dental bur dislodged in the maxillary antrum.
\end{abstract}

\section{KEYWORDS}

Maxillary sinusitis, dental bur.

\section{INTRODUCTION}

Dental foreign bodies are the most common cause of a unilateral maxillary sinusitis ${ }^{1}$. The presence of FB inside the maxillary antrum often results from the introduction of material through an oroantral communication or from trauma ${ }^{2}$. The route of penetrating foreign bodies can be transfacial, transnasal, transorbital and transoral ${ }^{3}$. Different types of materials presented in the maxillary sinus such as amalgam fillings, parts of a tooth, root fragments, gutta-percha, silver cone, impression materials, food debris and dental implants. ${ }^{4-6}$ Literature review revealed only a few cases of iatrogenic DB lodged into the maxillary sinus. ${ }^{4,7}$

\section{CASE REPORT}

A 63 years old male patient complaining from continuous mild to moderate pain and heaviness in the left cheek region. The patient underwent dental treatment two years ago, where the left upper second molar had been extracted. After seven days, the adjacent second premolar and first molar were prepared to receive two ceramic crowns; the patient gave a history of pain and discomfort after that. No relevant findings on both extra- and intra-oral examinations with no signs or symptoms of an oroantral fistula. There was no history of systemic diseases. Panoramic view and computed tomography (CT) showed a metal FB (DB) in the left maxillary antrum with the signs of chronic inflammation. The bur was embedded in the thick mucus tissue of the sinus (Fig. 1).
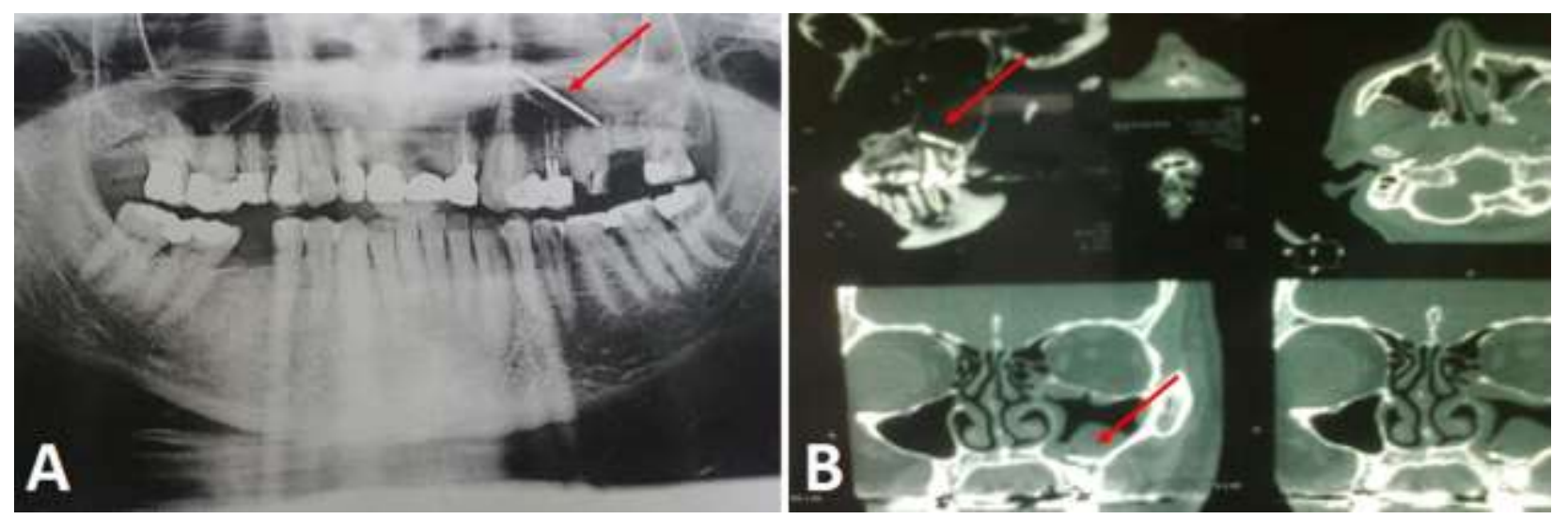

FIGURE 1. Preoperative imaging: (A) Orthopantomogram showing metallic FB (red arrow) in the left maxillary antrum. (B) CT-scans showing metallic FB (red arrow) in the left maxillary antrum with signs of chronic inflammation. 
The patient informed of the findings, the treatment plan was to remove the FB by a Caldwell-Luc surgical approach. The procedure carried out under local anesthesia, a mucoperiosteal flap of a semilunar type was raised extending from the canine fossa to the upper second molar area, creating a window through the lateral bony wall of the sinus cutting through the Schneiderian membrane. Meticulous curettage of the chronically inflamed tissue along with copious irrigation of the area with normal saline was performed to remove the FB, which was a DB $10 \mathrm{~mm}$ in length (Fig. 2). A one-year post-operative panoramic view showed the very well-healed sinus with no signs or symptoms of sinusitis (Fig. 3).
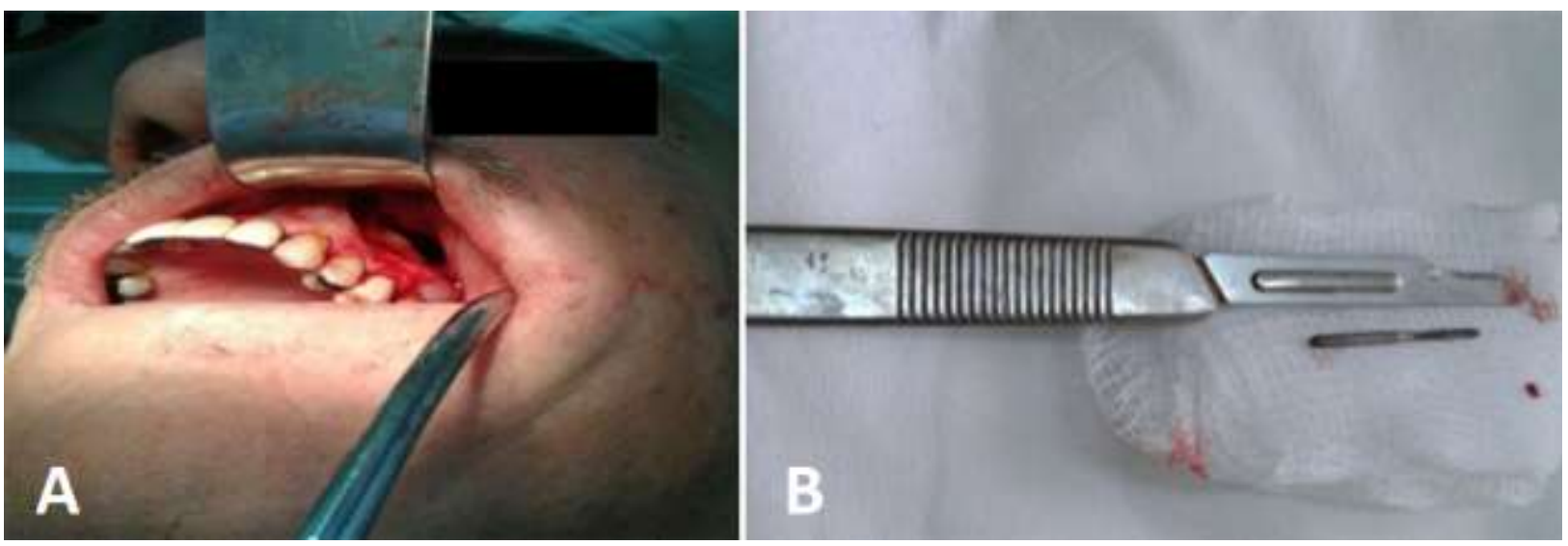

FIGURE 2. (A) Caldwell-Luc approach to the left maxillary sinus. (B) Dental Bur delivered from the left maxillary sinus.

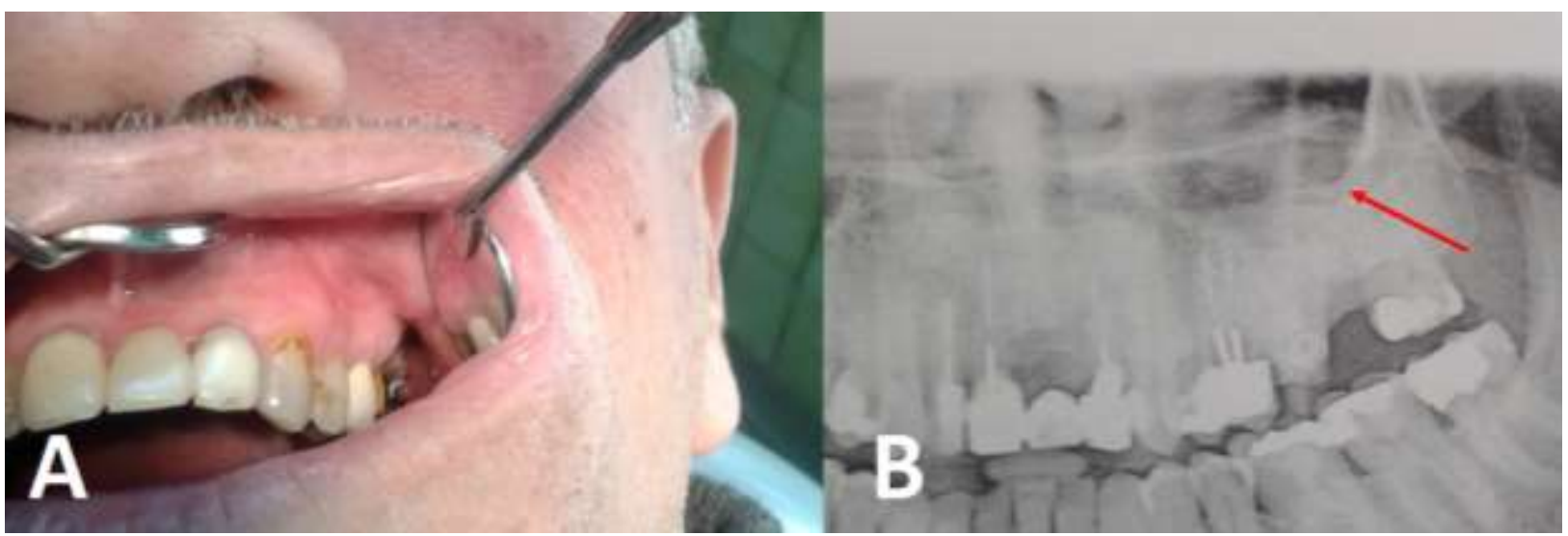

FIGURE 3. (A) Clinical picture of the site of operation after six months. (B) Post-operative Orthopantomogram after one year showing very well-healed left maxillary sinus (red arrow).

\section{DISCUSSION}

The incidence of FB inside the maxillary antrum is a rare condition, mostly occurring during trauma. Dental treatment may also introduce foreign bodies into the sinus especially during surgical extraction of upper molars or during insertion of dental implant. ${ }^{4,5}$

Displacement of DB into maxillary antrum are infrequently seen. The iatrogenic dislodgment of a DB is very rare with only six reported cases in the literature. ${ }^{4,7}$ These FBs may not necessarily symptomatic; the main complaint of this case was discomfort or heaviness in the cheek. Foreign bodies in the antrum could be discovered during a routine examination without any associated signs or symptoms of chronic inflammation, others presented with severe inflammation extending to the ethmoid sinus. Symptoms of odontogenic and nonodontogenic sinusitis are usually the same. ${ }^{8,9}$

Various complications due to FBs in the sinus were reported in the literature, which could be potentially serious sequelae including chronic sinusitis, aspergillosis, meningitis. ${ }^{3}$ 
The absence of oroantral fistula does not rule out the presence of FB in the antrum, as the case in this study, where the upper second molar extracted without any complications. After one week and during the preparation of the adjacent teeth to receive the crowns, the bur entered through the socket of the extracted upper second molar; the bur ejected from high-speed handpiece (mostly turbine) due to poor retention. The patient should be notified, for ethical and legal issues. It should always be considered the possibility of odontogenic sinusitis when the patient presented with unilateral symptoms. In such cases, a preoperative CT scan is necessary to know the exact site, size, nature, and the number of foreign bodies. ${ }^{10}$, 11

The Caldwell-Luc technique is the most widely used method to remove FB from the sinus. Some authors prefer to remove the FB by an endoscopic procedure, however, it needs operator experience, large FBs cannot be retrieved by endoscopy. In addition to that, the FB associated with chronic inflammation embedded in thick mucosal tissue is not easily removed by endoscopy except when a newly lodged object is removed through sinus ostium by endoscope

\section{CONCLUSION}

The review of this case report highly recommends delaying tooth preparation or any invasive dental procedures immediately after tooth extraction or during the first two weeks because the socket is still fresh. Tooth sectioning during extraction also carries the same risk of bur penetration. Whenever the patient complaining from unilateral sinusitis, always suspect and seek if there is FB in the symptomatic sinus. Whenever FB is present, it should be removed to prevent chronic sinusitis and its serious complications.

\section{REFRENCES}

1- 1 Tingsgaard PK, Larsen PL. [Chronic unilateral maxillary sinusitis caused by foreign bodies in the maxillary sinus]. Ugeskr Laeger. 1997 Jul 7;159(28):4402-4.

2- Sahin YF, Muderris T, Bercin S, et al. Chronic maxillary sinusitis associated with an unusual foreign body: a case report. Case Rep Otolaryngol. 2012;2012:903714.

3- Lee KC, Lee SJ. Clinical features and treatments of odontogenic sinusitis. Yonsei Med J. 2010 Nov;51(6):9327.

4- Smith JL 2nd, Emko P. Management of a maxillary sinus foreign body (dental bur). Ear Nose Throat J. 2007 Nov;86(11):677-8.

5- Tsodoulos S, Karabouta I, Voulgaropoulou M, et al. Atraumatic removal of an asymptomatic migrated dental implant into the maxillary sinus: a case report. J Oral Implantol. 2012 Apr;38(2):189-93.

6- Liston PN, Walters RF. Foreign bodies in the maxillary antrum: a case report. Aust Dent J. 2002 Dec;47(4):344-6.

7- Abe K, Beppu K, Shinohara M, et al. An iatrogenic foreign body (dental bur) in the maxillary antrum: a report of two cases. Br Dent J. 1992 Jul 25;173(2):63-5.

8- Crovetto-Martínez R, Martin-Arregui FJ, Zabala-López-deMaturana A, et al. Frequency of the odontogenic maxillary sinusitis extended to the anterior ethmoid sinus and response to surgical treatment. Med Oral Patol Oral Cir Bucal. 2014 Jul 1;19(4):e409-13.

9- Simuntis R, Kubilius R, Vaitkus S. Odontogenic maxillary sinusitis: a review. Stomatologija. 2014;16(2):39-43.

10- W. Pawlak, Kaczkowski H. Unusual Foreign Body Presenting in Maxillary Sinus - Case Report. Dent. Med. Probl. 2006;43(2):305-8.

11- Mehra P, Murad H. Maxillary sinus disease of odontogenic origin. Otolaryngol Clin North Am. 2004 Apr;37(2):347-64. 